\title{
A model of car-following behavior at sags
}

B. Goñi Ros, V.L. Knoop, W.J. Schakel, B. van Arem, S.P. Hoogendoorn

25th September 2013

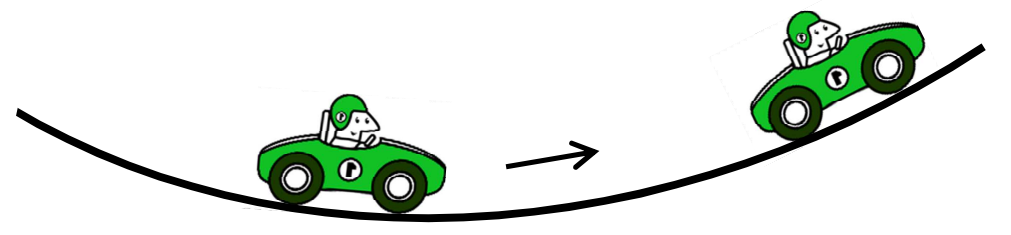

Jülich Supercomputing Centre

Traffic and Granular Flow '13 


\section{Outline}

1. Background

2. Car-following model

3. Empirical data

4. Simulation study

5. Conclusions 


\section{Background}

\section{What is a sag?}

- Sag: freeway section along which the gradient changes significantly from downwards to upwards

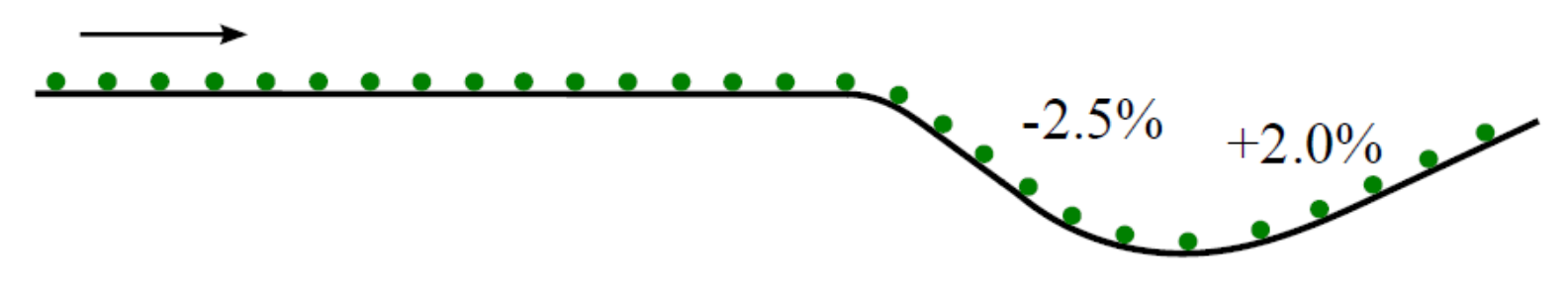




\section{Background}

\section{What is car-following behavior?}

Vehicle $n \quad$ Vehicle $n-1$

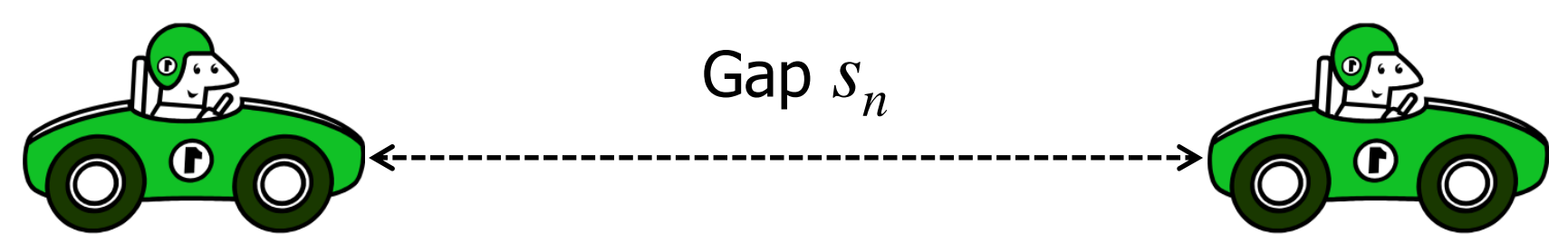

Speed $v_{n}$

Speed $v_{n-1}$

Relative speed $\Delta v_{n}=v_{n-1}-v_{n}$

Acceleration $d v_{n} / d t=f\left(s_{n}, v_{n}, \Delta v_{n}, \ldots\right)$

Equilibrium traffic conditions

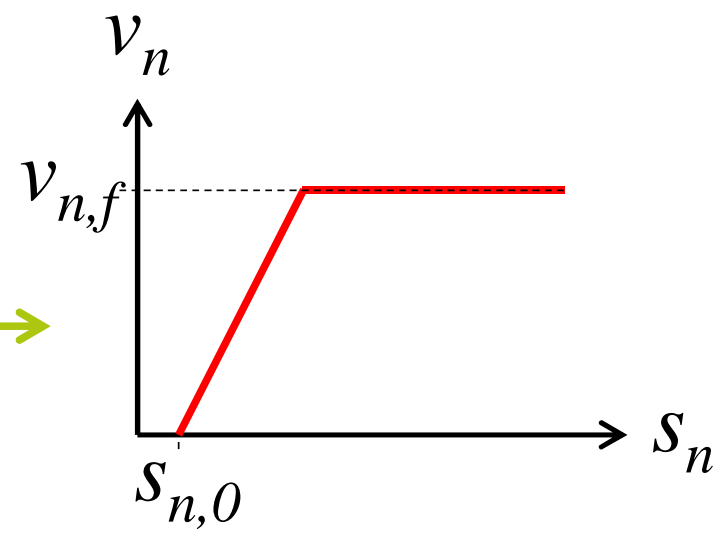




\section{Background}

\section{Car-following behavior at sags}

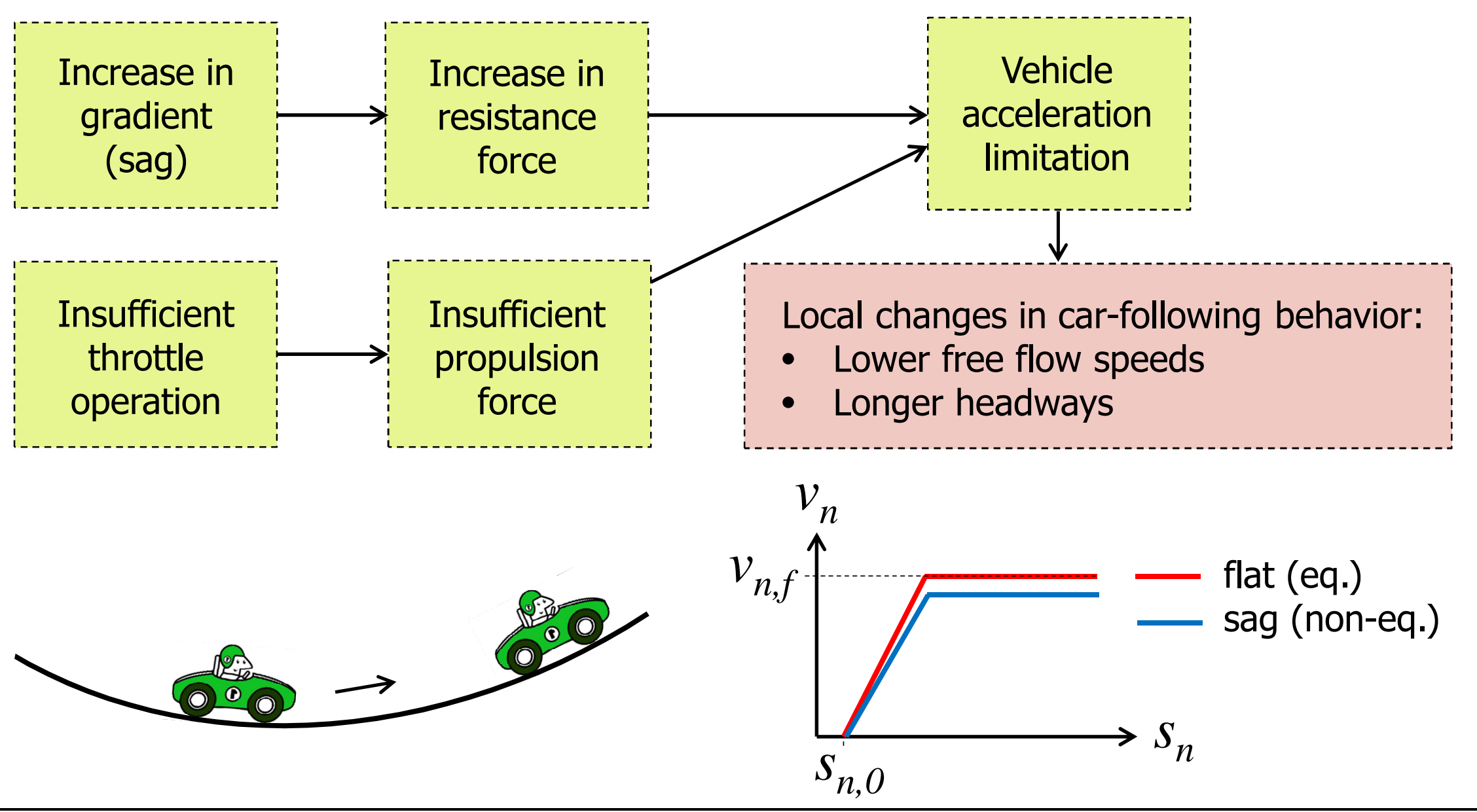




\section{Background}

\section{Sags become freeway bottlenecks}

- Those changes in car-following behavior have a negative influence on freeway capacity

$>$ Capacity is $10-20 \%$ lower at sags than at flat sections

- In conditions of high demand, traffic breaks down at sags $\rightarrow$ capacity drop
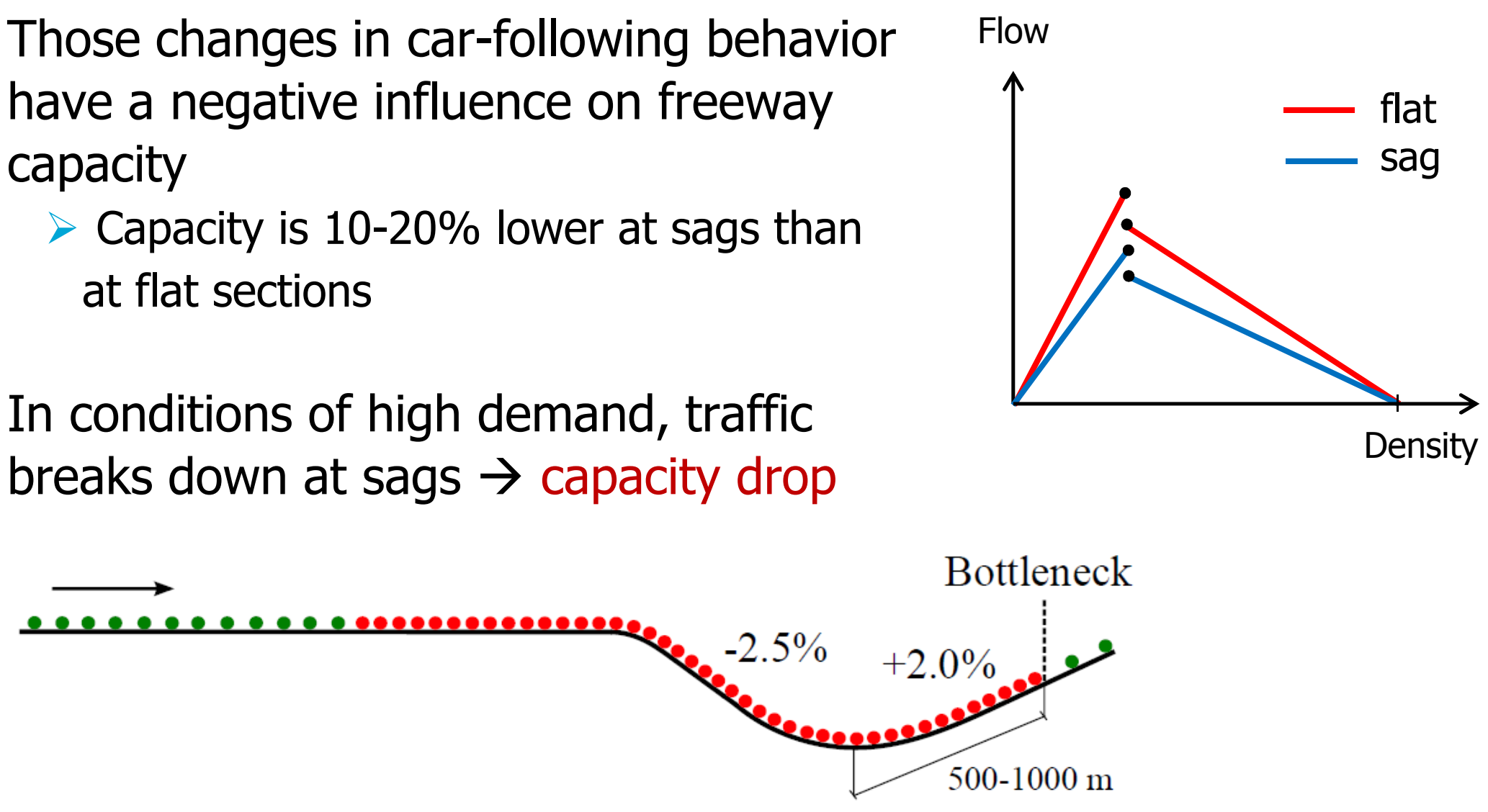


\section{Car-following model}

\section{Influence of gradient: principles}

1. Drivers perceive the change in gradient at sags

2. Drivers are not able to fully compensate for the increase in resistance force at the beginning of the uphill section

$>$ Limiting effect on vehicle acceleration

3. Along the uphill section, drivers are able to gradually compensate for the increase in resistance force

$>$ The limiting effect on vehicle acceleration decreases over time/space 


\section{Car-following model}

Formulation

- Acceleration: $\dot{v}(t)=f_{1}(t)+f_{2}(t)$

- First term: influence of speed, gap and relative speed $(\approx$ IDM)

$$
\begin{aligned}
& f_{1}(t)=a \cdot\left[1-\left(\frac{v(t)}{v_{\text {des }}(t)}\right)^{4}-\left(\frac{s^{*}(t)}{s(t)-l}\right)^{2}\right] \\
& \text { where: } s^{*}(t)=s_{0}+v(t) \cdot T(t)+\frac{v(t) \cdot \Delta v(t)}{2 \cdot \sqrt{a b}} \\
& \underset{\substack{\text { Safe time } \\
\text { gap (s) }}}{ } \quad T(t)= \begin{cases}T_{0} & \text { if } v(t) \geq v_{\text {crit }} \\
\gamma \cdot T_{0} & \text { if } v(t)<v_{\text {crit }}\end{cases}
\end{aligned}
$$

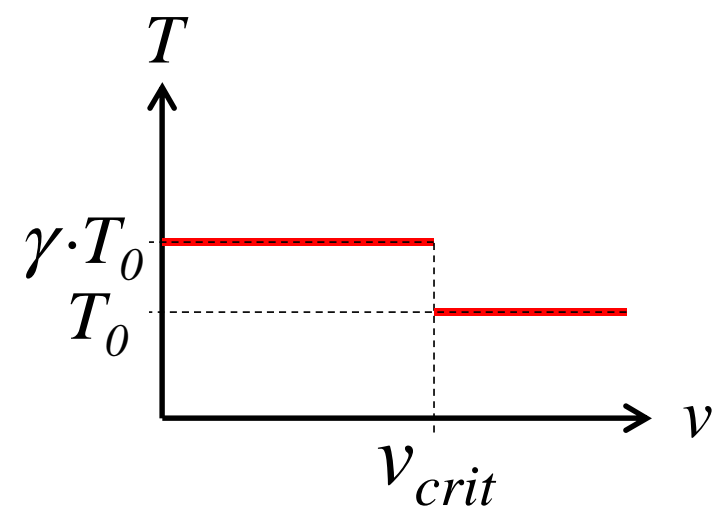




\section{Car-following model}

Formulation

- Acceleration: $\dot{v}(t)=f_{1}(t)+f_{2}(t)$

- Second term: influence of gradient

$$
\begin{aligned}
f_{2}(t)= & -g \cdot\left[G(t)-G_{c}(t)\right. \\
\text { where: } & G_{c}(t)= \begin{cases}G(t) \\
G\left(t_{c}\right)+c \cdot\left(t-t_{c}\right) & \text { if } G(t)>G\left(t_{c}\right)+c \cdot\left(t-t_{c}\right)\end{cases} \\
& t_{c}=\max \left(t \mid G_{c}(t)=G(t)\right)
\end{aligned}
$$
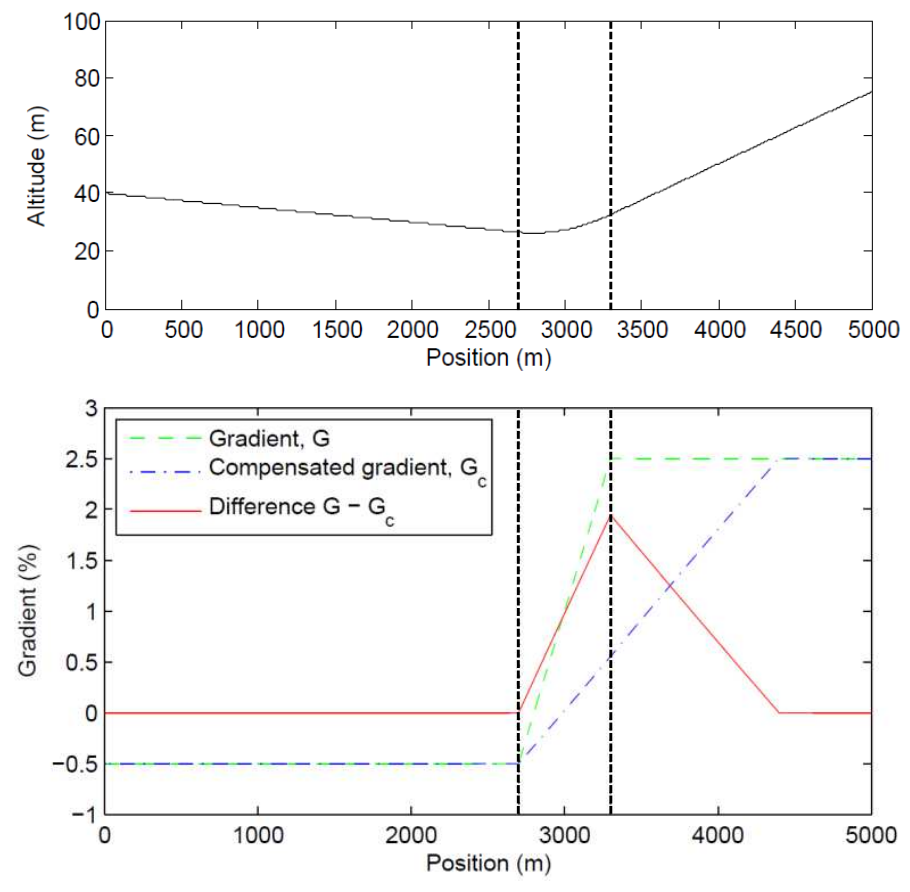


\section{Empirical data}

\section{Study site}

- Yamato sag, Tomei Expressway (Japan)

- 3 lanes
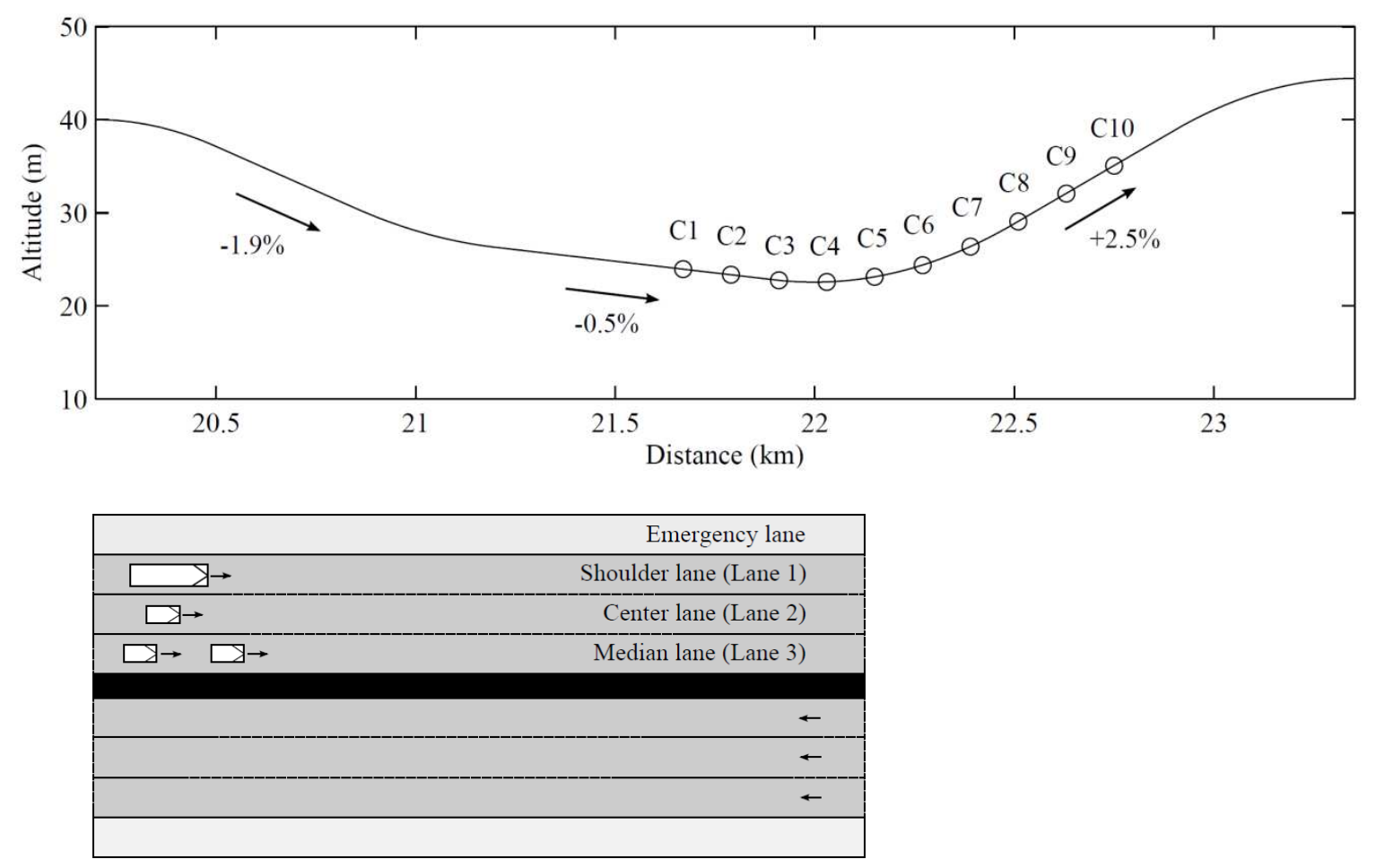


\section{Empirical data}

Speed and flow data

- Bottleneck location (head of the queue):

$>\mathrm{x} \approx 22.5 \mathrm{~km}$, $500 \mathrm{~m}$ downstream of the bottleneck (camera 8)

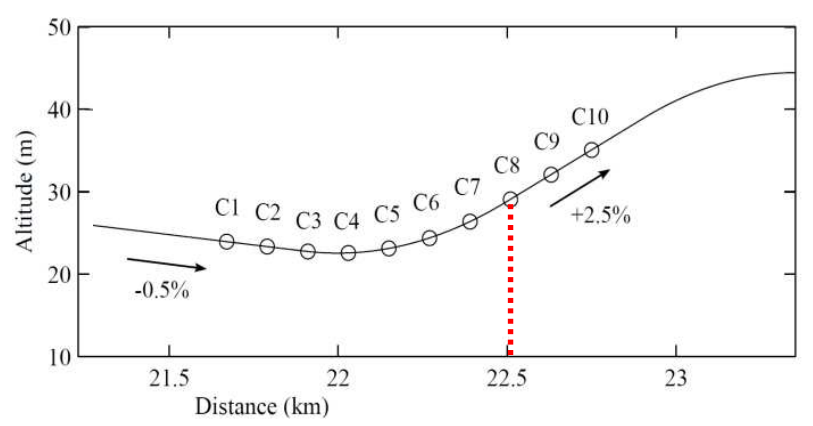

$x=22.5 \mathrm{~km}$ (bottleneck)
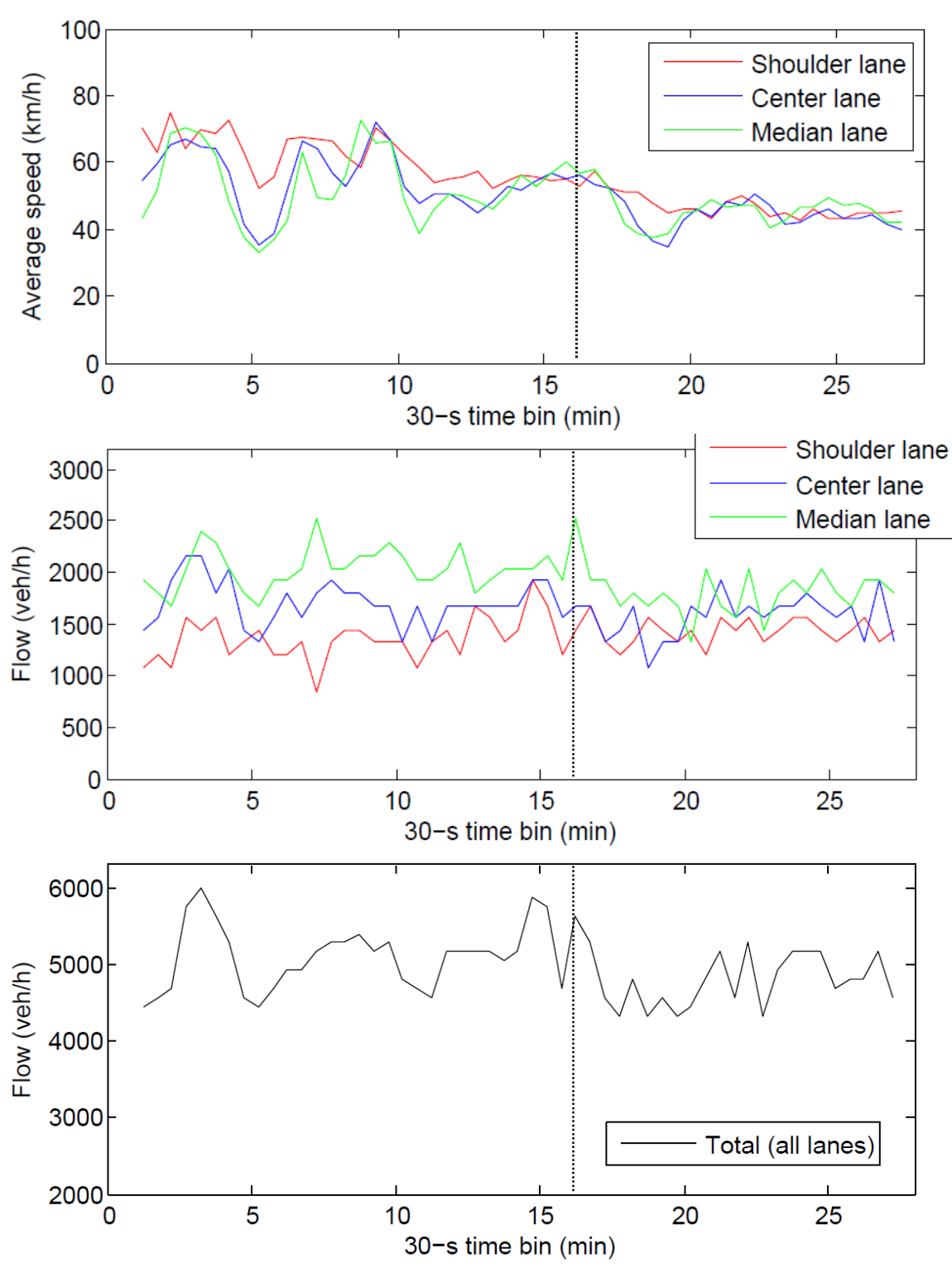


\section{Simulation study}

Set-up

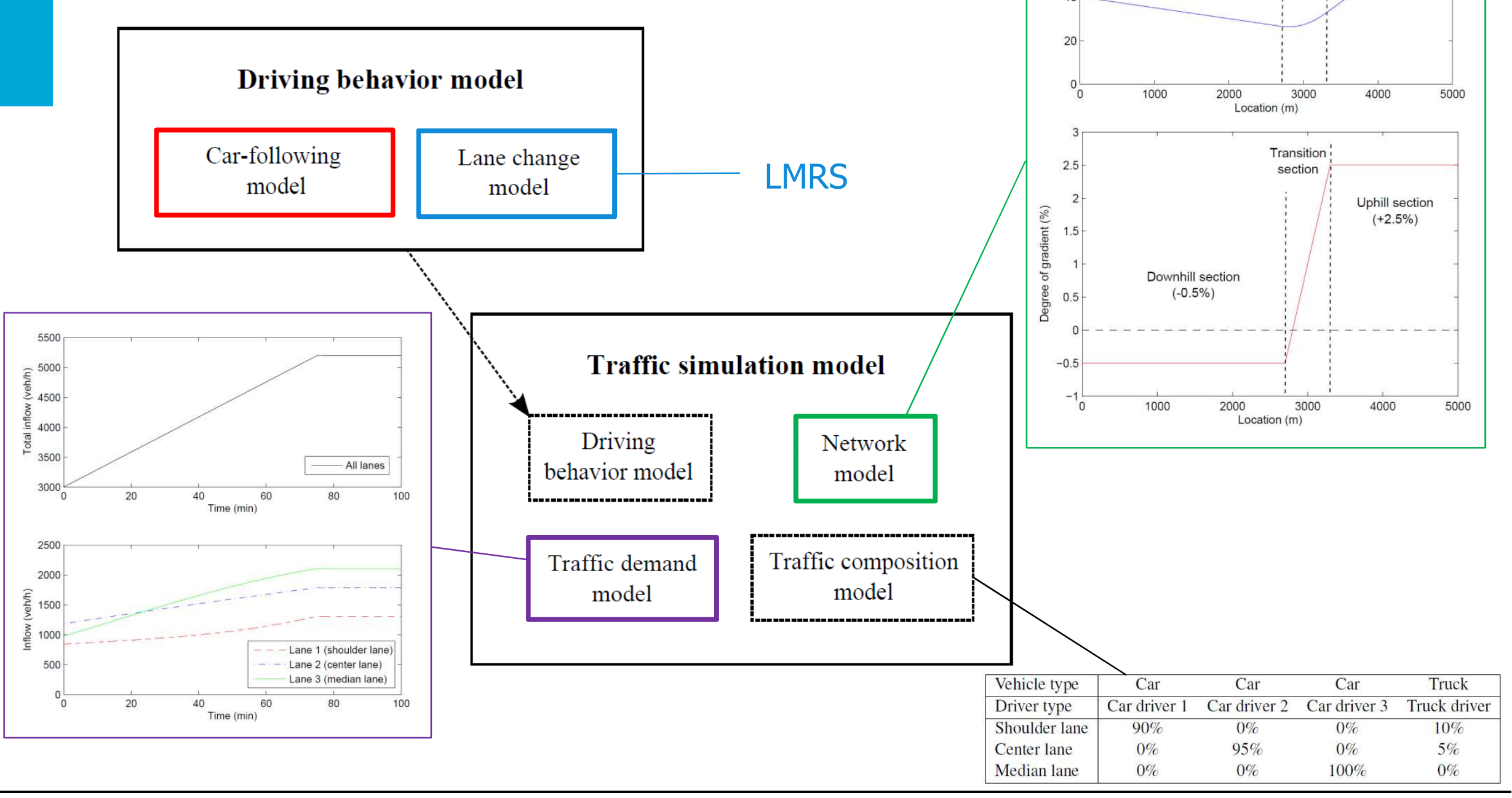




\section{Simulation study}

\section{Results}
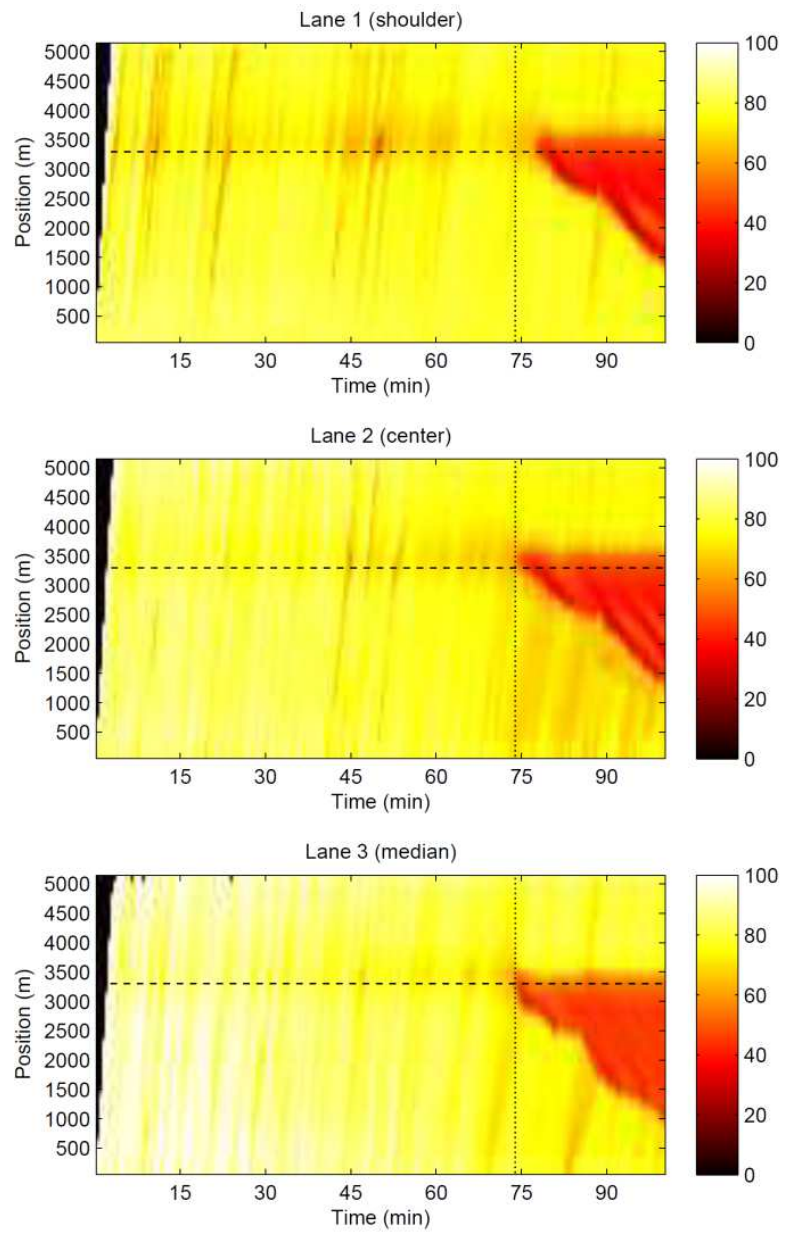

$\mathrm{x}=3200 \mathrm{~m}$ (bottleneck)
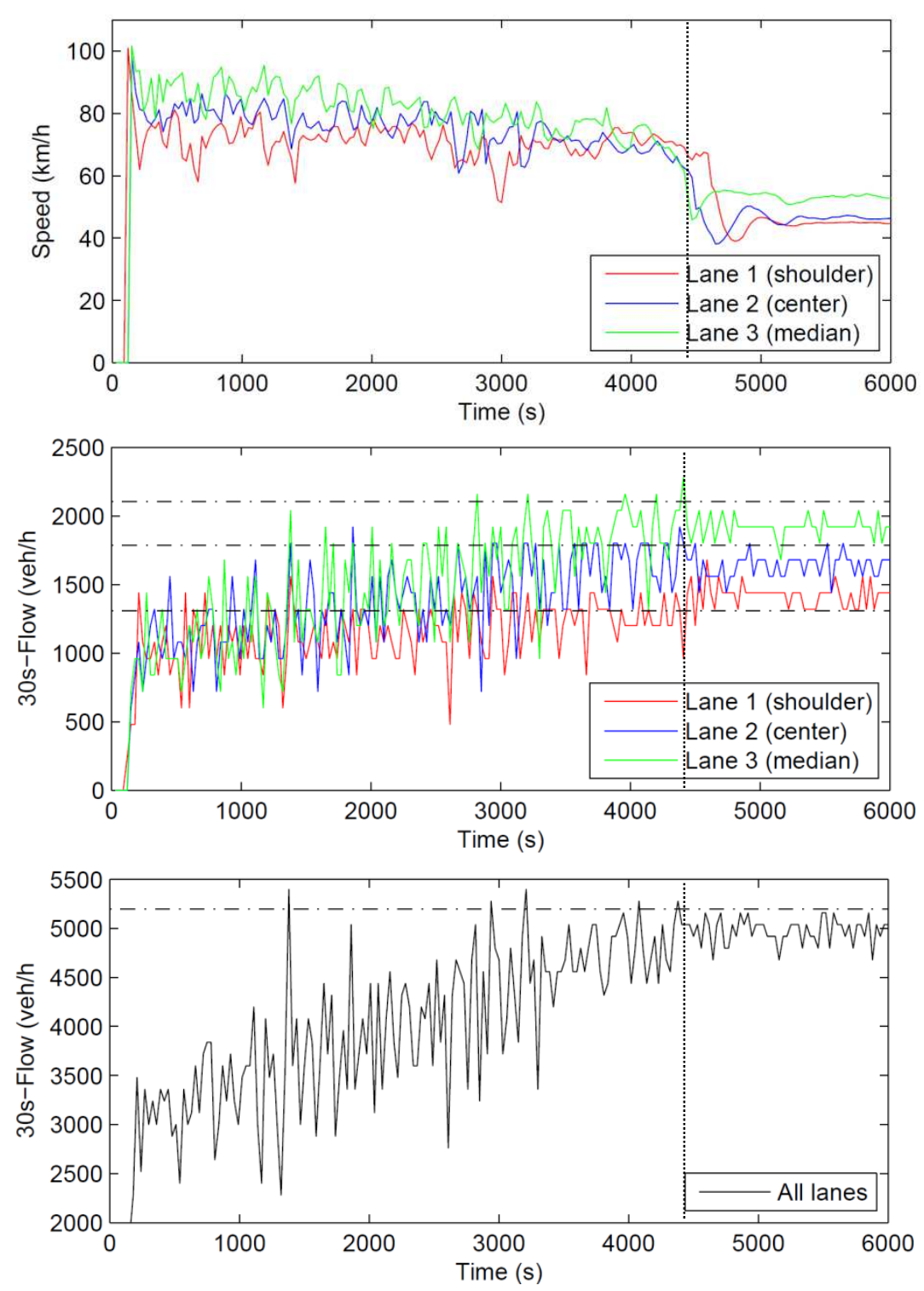

\section{TUUDelft $\oplus$ TOYOTA}




\section{Conclusions}

- New car-following model that takes into account the influence of gradient on vehicle acceleration

- Formulation: IDM + 1 additional parameter

- Key phenomena reproduced by our model:

$\checkmark$ Vehicle acceleration limitation

$\checkmark$ Reduced capacity

$\checkmark$ Bottleneck location

$\checkmark$ Capacity drop in congestion

- Use of the model to test the effectiveness of control measures to mitigate congestion at sags 


\section{Any questions? b.goniros@tudelft.nl}




\section{Parameters}

\begin{tabular}{|l|cccc|}
\hline Vehicle type & Car & Car & Car & Truck \\
\hline Driver type & Car driver 1 & Car driver 2 & Car driver 3 & Truck driver \\
\hline$l(\mathrm{~m})$ & 4 & 4 & 4 & 15 \\
$a_{0}\left(\mathrm{~m} / \mathrm{s}^{2}\right)$ & 1.25 & 1.25 & 1.25 & 0.50 \\
$b_{0}\left(\mathrm{~m} / \mathrm{s}^{2}\right)$ & 1.80 & 1.80 & 1.80 & 1.50 \\
$T_{0}(\mathrm{~s})$ & 1.45 & 1.20 & 1.15 & 1.50 \\
$s_{0}(\mathrm{~m})$ & 3 & 3 & 3 & 3 \\
$v_{\text {lim }}(\mathrm{km} / \mathrm{h})$ & 100 & 100 & 100 & 85 \\
$v_{\text {max }}(\mathrm{km} / \mathrm{h})$ & 150 & 150 & 150 & 100 \\
$v_{\text {crit }}(\mathrm{km} / \mathrm{h})$ & 60 & 60 & 60 & 60 \\
$c_{0}\left(\mathrm{~s}^{-1}\right)$ & 0.00042 & 0.00042 & 0.00042 & 0.00042 \\
$\gamma(-)$ & 1.15 & 1.15 & 1.15 & 1.15 \\
$\bar{\delta}(-)$ & 0.92 & 0.97 & 1.03 & 1.00 \\
$\sigma_{\delta}(-)$ & 0.03 & 0.10 & 0.10 & 0.00 \\
$\bar{v}_{\text {des }, t}(\mathrm{~km} / \mathrm{h})$ & & & & 85 \\
$\sigma_{v_{\text {des }, t}}(-)$ & & & & 2.5 \\
\hline$T_{\text {min }, 0}(\mathrm{~s})$ & 0.56 & 0.56 & 0.56 & 0.56 \\
$\tau(\mathrm{s})$ & 25 & 25 & 25 & 25 \\
$x_{0}(\mathrm{~m})$ & 200 & 200 & 200 & 200 \\
$v_{\text {gain }}(\mathrm{km} / \mathrm{h})$ & 70 & 50 & 50 & 70 \\
$d_{\text {free }}^{i j}(-)$ & 0.365 & 0.365 & 0.365 & 0.365 \\
$d_{\text {sync }}^{i j}(-)$ & 0.577 & 0.577 & 0.577 & 0.577 \\
$d_{\text {coop }}^{i j}(-)$ & 0.788 & 0.788 & 0.788 & 0.788 \\
\hline
\end{tabular}

